The Chinese Society of Theoretical and Applied Mechanics

Chinese Journal of Mechanics Press, Beijing, China

Allerton Press, INC., New York, U.S.A.

\title{
NUMERICAL AND EXPERIMENTAL INVESTIGATION OF FLOW OVER A SEMICIRCULAR WEIR
}

\author{
Liu Chunrong (刘春嵘) Huhe Aode (呼和敖德) Ma Wenju (马文驹) \\ (Institute of Mechanics, Chinese Academy of Sciences, Beijing 100080, China)
}

\begin{abstract}
The water flow over a semicircular weir is investigated numerically and experimentally in this paper. The numerical model solves the Reynolds equation for a mean flow field with the $k-\varepsilon$ turbulent model. To trace the motion of the free surface, the VOF method with geometric reconstruction is employed. The velocity of the flow is measured by means of LDV technique. Four types of flow patterns, the position of the separation and reattachment point, the distribution of shear stress on the bed at downstream of the weir are presented and discussed. The numerical results agree well with the experiment data.
\end{abstract}

KEY WORDS: flow over semicircular weir, numerical modeling, VOF method, shear stress, LDV technique

\section{INTRODUCTION}

Semicircular weir is a new type of construction developed in 1990's. It has many advantages compared with other types of weirs. So this type of weir is used in hydraulic engineering recently. It is very important to investigate the behaviors of the flow over the weir, the bottom shear stress and the local scour around the semicircular weir.

There are a number of numerical studies on water flow over obstacles, most of which are based on the inviscid model (Lamb ${ }^{[1]}$, Forbes and Schwartz ${ }^{[2]}$, Dias and Broeck ${ }^{[3]}$ ). For the condition of small deformed free surface, the motion of the free surface can be simulated by an inviscid model. When the free surface undergoes a large deformation with turbulence, the inviscid model is invalid. The method of Volume of Fluid (VOF) ${ }^{[4]}$ developed in 1980's is suitable to simulate the condition of large deformed free surface. This method is used to investigate the hydraulic jump and breaking waves ${ }^{[5,6]}$. There are also some experimental studies on the flow over the weir ${ }^{[7]}$. Most of them considered a simple geometrical shape and rectangle meshes were employed. There is little investigation on the flow over a semicircular weir, and scarcely any detailed and systematical work that studied the flow patterns over a semicircular weir, the separation and reattachment of the flow over the weir and the distribution of the bottom shear stress.

In this paper the turbulence flow over a semicircular weir is investigated. In order to adapt the geometrical shape of the boundary, the unstructured mesh is employed. The method of VOF is adopted to trace the free surface. For the unstructured mesh, the shape of the free surface is difficult to simulate accurately. In this paper the geometric reconstruction

Received 11 January 2001, revised 11 December 2001 
approach is used to improve the accuracy of the calculation. The standard $k-\varepsilon$ model is used for the turbulence closure. Four types of flow patterns: surface jet; surface wave; plunging jet and hydraulic jump are presented in the paper. The effects of Reynolds number Re, Froude number $\mathrm{Fr}$ and the ratio of weir height to water depth $D / H$ on the flow patterns are discussed. The position of the separation and reattachment of the flow over the weir and the bottom shear stress at downstream of the weir are also studied and discussed. The flow patterns have been visualized in water tunnel and the flow velocity at downstream of the weir are measured by means of LDV method. The bottom shear stress is calculated according to the velocity profile. The numerical results are compared with the experimental results.

\section{NUMERICAL MODELING}

\subsection{Governing Equations}

The governing equations are the unsteady incompressible two-dimensional continuity equation and Reynolds-averaged Navier-Stokes equation.

$$
\begin{aligned}
& \frac{\partial \rho}{\partial t}+\frac{\partial}{\partial x_{i}}\left(\rho u_{i}\right)=0 \\
& \frac{\partial}{\partial t} \rho u_{j}+\frac{\partial}{\partial x_{i}} \rho u_{i} u_{j}=-\frac{\partial P}{\partial x_{j}}+\frac{\partial}{\partial x_{i}}\left(\mu+\mu_{t}\right)\left(\frac{\partial u_{i}}{\partial x_{j}}+\frac{\partial u_{j}}{\partial x_{i}}\right)+\rho g_{j}
\end{aligned}
$$

where $u_{i}$ are the velocity components $U_{x}, U_{y}$; the density $\rho=\alpha_{\mathrm{A}} \rho_{\mathrm{A}}+\alpha_{\mathrm{W}} \rho_{\mathrm{W}}, \alpha_{\mathrm{A}}$ and $\alpha_{\mathrm{W}}$ are the volume fraction of air and water, respectively; $\rho_{\mathrm{A}}$ and $\rho_{\mathrm{W}}$ are the density of air and water, respectively; $P$ is the pressure; $g_{j}$ is the gravity. The viscosity $\mu=\alpha_{\mathrm{A}} \mu_{\mathrm{A}}+\alpha_{\mathrm{W}} \mu_{\mathrm{W}}$, $\mu_{\mathrm{A}}$ and $\mu_{\mathrm{W}}$ are the viscosity of air and water, respectively; $\mu_{t}$ is the eddy viscosity and can be expressed as

$$
\mu_{t}=\rho C_{u} \frac{k^{2}}{\varepsilon}
$$

These equations are coupled with the standard $k-\varepsilon$ turbulent model

$$
\begin{aligned}
& \rho \frac{\mathrm{d} k}{\mathrm{~d} t}=\frac{\partial}{\partial x_{i}}\left[\left(\mu+\frac{\mu_{t}}{\sigma_{k}}\right) \frac{\partial k}{\partial x_{i}}\right]+G_{k}-\rho \varepsilon \\
& \rho \frac{\mathrm{d} \varepsilon}{\mathrm{d} t}=\frac{\partial}{\partial x_{i}}\left[\left(\mu+\frac{\mu_{t}}{\sigma_{\varepsilon}}\right) \frac{\partial \varepsilon}{\partial x_{i}}\right]+C_{\varepsilon 1} \frac{\varepsilon}{k} G_{k}-C_{\varepsilon 2} \rho \frac{\varepsilon^{2}}{k}
\end{aligned}
$$

where $k$ is the turbulent kinetic energy, $\varepsilon$ the turbulent dissipation rate. The empirical coefficients in the turbulent model are recommended: $C_{u}=0.09, C_{\varepsilon 2}=1.92, \sigma_{k}=1.0$, $\sigma_{\varepsilon}=1.3$.

To trace the location of the free surface with large deformation the VOF method is used. The volume fraction equation is

$$
\frac{\partial \alpha_{\mathrm{A}}}{\partial t}+u_{i} \frac{\partial \alpha_{\mathrm{A}}}{\partial x_{i}}=0
$$

The region where $\alpha_{\mathrm{A}}=0$ is occupied by the water, and the area where $\alpha_{\mathrm{A}}=1$ is filled with the air. According to the value of $\alpha_{\mathrm{W}}$, the location of the free surface can be determined. 


\subsection{Boundary and Initial Conditions}

In this study a two-dimensional semicircular weir is considered. The computing domain is a rectangle of $50 D$ long and $2.2 D$ high, where $D$ is the weir height. The water depth $H$ varies from $1.15 D$ to $2 D$. Above the water is a zone of air. Four types of boundary conditions are considered:

The velocity inlet condition

$$
\begin{array}{lll}
U_{x}=U_{0} \quad U_{y}=0 & k=0.02 U^{2} \quad \varepsilon=\frac{C_{u} k^{3 / 2}}{l} \quad l=0.07 H \\
\alpha_{\mathrm{A}}=0(0 \leq y \leq H) & \alpha_{\mathrm{A}}=1(H \leq y \leq 2.2 D) & \text { at } \quad x=-25 D
\end{array}
$$

The outer flow condition

$$
\frac{\partial \Phi}{\partial t}+U_{x} \frac{\partial \Phi}{\partial x}=0 \quad \text { at } x=25 D
$$

where $\Phi$ represents $U_{x}, U_{y}, k, \varepsilon, \alpha_{\mathrm{A}}$.

The wall condition: the wall function is applied on the bottom and the surface of the weir.

The pressure inlet condition

$$
p=\text { constant } \quad \text { at } y=2.2 D
$$

The initial condition

$$
U_{x}=\text { constant } \quad U_{y}=0 \quad \text { in the entire computing domain }
$$

\section{NUMERICAL METHODS}

The governing equations are discretized by a control-volume-based technique with an unstructured mesh. The discretization of Eqs.(2), (4), (5) on a given cell can be written as

$$
\rho V\left(\frac{\Phi^{n+1}-\Phi^{n}}{\Delta t}\right)+\sum_{f}^{N_{\text {faces }}} J_{f} \Phi_{f}=\sum_{f}^{N_{\text {faces }}} \Gamma_{\Phi}(\nabla \Phi)_{n} A_{f}+\sum_{f}^{N_{\text {faces }}} \delta_{u \Phi} p_{f} \boldsymbol{A}_{f} \cdot \boldsymbol{n}_{u}+S V
$$

where $N_{\text {faces }}$ is the number of faces enclosing the cell, $\Phi$. represents $U_{x}, U_{y}, k, \varepsilon$ at cell centers ( $c_{0}$ and $c_{1}$ in Fig.1), $\Phi_{f}$ is the value convected through face $f, J_{f}$ is the mass flow rate through face $f, \Gamma_{\Phi}$ is the diffusion coefficient, $(\nabla \Phi)_{n}$ is the magnitude of $\nabla \Phi$ normal to face $f, p_{f}$ is the pressure at face $f, n_{u}$ is unit vector which has the same direction with flow velocity, $\boldsymbol{A}_{f}$ is the surface area vector, $A_{f}$ is the area of face $f\left(A_{f}=\left|\boldsymbol{A}_{f}\right|\right), V$ is the cell volume, $S$ is the source term. $\delta_{u \Phi}=1$ when $\Phi$ represents $U_{x}, U_{y} ; \delta_{u \Phi}=0$ when $\Phi$ represents $k, \varepsilon$. Equation (7) can be linearized as

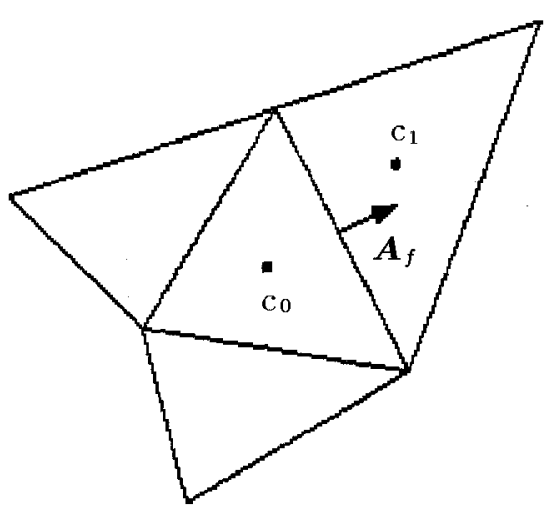

Fig.1 Cells used to illustrate discretization of governing equations 


$$
a_{\mathrm{p}} \Phi=\sum_{\mathrm{nb}} a_{\mathrm{nb}} \Phi_{\mathrm{nb}}+a
$$

where $\Phi_{\mathrm{nb}}$ is the value of $\Phi$ at the centers of the cells conjoint to the given cell, $a_{\mathrm{p}}$ and $a_{\mathrm{nb}}$ are the linearized coefficients for $\Phi$ and $\Phi_{\mathrm{nb}}$, and $a$ is the item which does not include $\Phi$.

The discretization of Eqs.(1) and (6) on a given cell can be written as

$$
\frac{\psi^{n+1}-\psi^{n}}{\Delta t} V+\sum_{f}^{N_{\text {faces }}} U_{n} \psi^{n}=0
$$

$\psi$ represents $\rho, \alpha_{\mathrm{A}}, U_{n}$ is the velocity components normal to face $f$.

In order to consider the pressure-velocity coupling, it is necessary to relate the face values of velocity to the values of velocity at the cell centers. Linear interpolation of cellcentered velocities to the face results in an unphysical checker-boarding of pressure. A method similar to that outlined by Rhie and Chow ${ }^{[8]}$ is employed. The mass flow rate through face $J_{f}$ may be written as

$$
J_{f}=\overline{J_{f}}+d_{f}\left(p_{\mathrm{c}_{0}}-p_{\mathrm{c}_{1}}\right)
$$

where $p_{c_{0}}$ and $p_{\mathrm{c}_{1}}$ is the pressure with the cells on either side of the face, and $\overline{J_{f}}$ contains the influence of velocities in these cells (see in Fig.1), $d_{f}$ is a weighting factor. If the momentum equation is solved with a guessed pressure field $p^{*}$, the mass flow rate through face $J_{f}^{*}$ can be computed from Eq.(10). In order to satisfy the continuity equation, a correction $J_{f}^{\prime}$ is added to $J_{f}^{*}$ so that

$$
J_{f}=J_{f}^{*}+J_{f}^{\prime}
$$

$J_{f}^{\prime}$ can be written as

$$
J_{f}^{\prime}=d_{f}\left(p_{\mathrm{c}_{0}}^{\prime}-p_{\mathrm{c}_{1}}^{\prime}\right)
$$

where $p^{\prime}$ is the cell pressure correction. Substituting Eqs.(11) and (12) into the discrete continuity Eq.(9), the discrete equation for the pressure correction $p^{\prime}$ can be obtained as

$$
b_{\mathrm{p}} p^{\prime}=\sum_{\mathrm{nb}} b_{\mathrm{nb}} p_{\mathrm{nb}}^{\prime}+b
$$

where $b_{\mathrm{p}}$ and $b_{\mathrm{nb}}$ are the linearized coefficients for $p^{\prime}$ and $p_{\mathrm{nb}}^{\prime}$, and $b$ is the item which does not include $p^{\prime}$. The corrected pressure field can be obtained by solving Eq.(13). When corrected pressure filed is known, the corrected velocity field can be obtained.

The method of VOF with geometric reconstruction is used to compute the location of the free surface. By solving Eq.(9), the volume fraction of air and water can be obtained. According to the volume fraction of air and water, the position of the free surface can be computed using a piecewise-linear approach. The convection and diffusion fluxes through the free surface are computed to satisfy the free surface boundary conditions.

The unstructured mesh is adopted in this paper. In the region near the weir the triangle mesh is employed, and far away from the weir the rectangle mesh is adopted. The mesh size is generally about $0.1 \mathrm{D}$, it is refined to $0.05 \mathrm{D}$ near the free surface and the weir, and is further refined to $0.025 \mathrm{D}$ at the region where the free surface undergoes large deformation. Near the wall the boundary layer mesh is used. The total number of the grid nodes is about 
15000. The time step is chosen to satisfy $C u=U \Delta t / \Delta x<0.1$, in which $U$ is the magnitude of the flow velocity.

\section{EXPERIMENT}

The experiment was carried out in a recirculating water tunnel of $15 \mathrm{~m}$ long, $0.5 \mathrm{~m}$ wide, $0.6 \mathrm{~m}$ deep. A semicircular cylinder with a radius of $4.5 \mathrm{~cm}$ and of $0.5 \mathrm{~m}$ long was placed along the width of the water tunnel at a distance of $10 \mathrm{~m}$ from the inlet. The mean flow velocity at far upstream is ranged from $5 \mathrm{~cm} / \mathrm{s}$ to $20 \mathrm{~cm} / \mathrm{s}$. The flow field was visualized by the particle trace method. The flow velocity was measured by the LDV technique.

\section{RESULTS AND DISCUSSION}

\subsection{Flow Patterns}

The flow over a weir has four types of flow patterns: surface jet, surface wave, plunging jet, and hydraulic jump. The present results show that the flow patterns have little altered with $R e$ in the range of our investigation parameters for fixed $F r_{\mathrm{u}}$ and $D / H$, so the flow patterns are predominated by $F r_{\mathrm{u}}$ and $D / H$, where $F r_{\mathrm{u}}=U_{\mathrm{u}} / \sqrt{g H}, R e=U_{\mathrm{u}} D / \nu, U_{\mathrm{u}}$ is the mean flow velocity at far upstream.

The relationship between flow patterns, $h / H$ ( $h$ is the water depth of the downstream) and $F r_{\mathrm{u}}, D / H$ is shown in Fig.2. Figure 2 shows that when $F r_{\mathrm{u}}$ or $D / H$ are small (region A), the water level at the downstream and the upstream of the weir is almost the same. The free surface is flat. This situation is called the surface jet (Figs.3(a), 3(b)). When $F r_{\mathrm{u}}$ or $D / H$ increases (region $\mathrm{B}$ ), the water level of the downstream is lower than that of the upstream. The flow pattern changes to the surface wave (Figs.3(c), 3(d)). For the conditions of the surface jet and surface wave, the higher velocity flow at the downstream of the weir is along the free surface. A clockwise rotating circulation is formed behind the weir. When $F r_{\mathrm{u}}$ or $D / H$ increases further (region $\mathrm{C}$ ), the flow becomes unstable. The flow pattern will change to plunging condition from the surface wave when a small perturbation exists. When $F r_{\mathrm{u}}$ or $D / H$ is large enough and $F r_{\mathrm{d}}<1\left(F r_{\mathrm{d}}=U_{\mathrm{d}} / \sqrt{g h}, U_{\mathrm{d}}\right.$ is the mean velocity of the downstream), the flow pattern is plunging jet (Figs.3(e), 3(f)). For this condition, the higher velocity flow goes from the surface of the weir to the bottom, two vortices are formed behind the weir, one is a clockwise rotating vortex at the corner of the

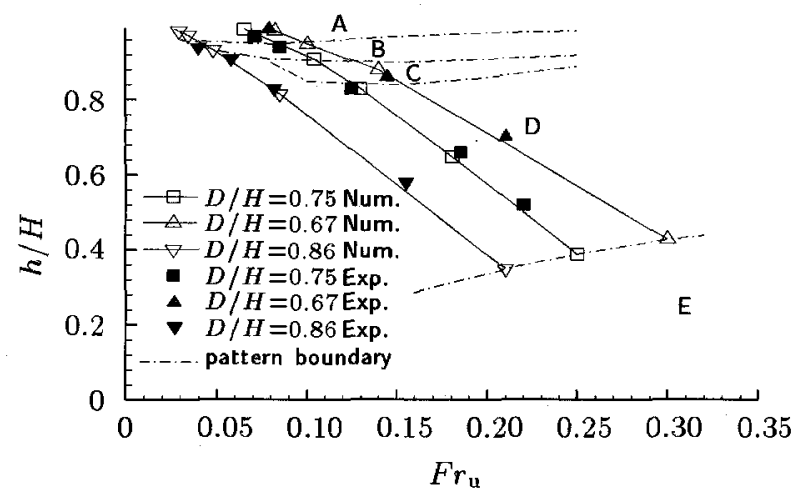

Fig.2 Diagram of the flow pattern regions 
weir and the bottom, the other is a counter-clockwise rotating vortex near the free surface. When $F r_{\mathrm{d}}>1$, the hydraulic jump will appear at the downstream. The flow patterns of surface jet, surface wave, plunging condition are obtained by numerical simulation and experiment, which are shown in Fig.3. It is shown that the numerical results agree well with the experiment.

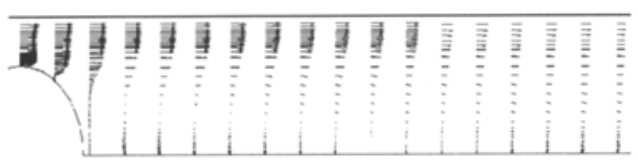

(a) Numerical

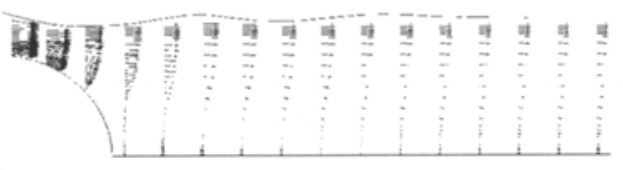

(c) Numerical

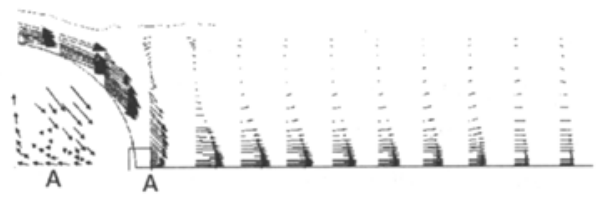

(e) Numerical

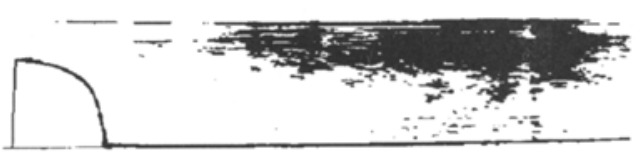

(b) Experimental

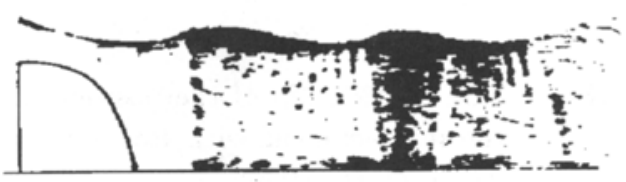

(d) Experimental

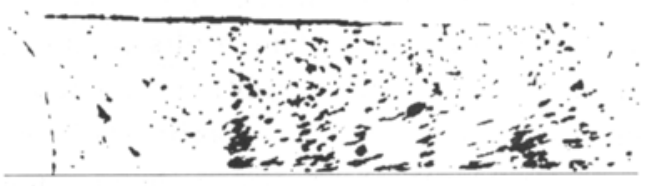

(f) Experimental

Fig.3 The flow patterns over the weir. (a) and (b) are surface jet: $F r_{\mathfrak{u}}=0.075, D / H=0.67$, $R e=2880 ;(\mathrm{c})$ and (d) are surface wave: $F r_{\mathrm{u}}=0.079, D / H=0.75, R e=2880$; (e) are (f) are plunging jet: $F r_{\mathbf{u}}=0.085, D / H=0.86, R e=2880$, time $=260 \mathrm{~s}$

\subsection{The Position of the Reattachment Point}

The boundary layer will be separated from the surface of the weir by the reverse pressure gradient, then it reattaches to the bottom at the downstream of the weir. The effects of the parameters $R e, F r_{\mathrm{u}}$ and $D / H$ on the position of the reattachment are studied in this paper.

For surface jet, the position of the reattachment is little affected by $F r_{\mathbf{u}}$ and $D / H$ when $R e$ remains constant. So it is predominated by $R e$, which is shown in Fig.4. Figure 4 shows that the distance from the position of the reattachment to the center of the weir, $L$, decreases fast as $R e$ increases for $R e<5 \times 10^{5}$, and the position of the reattachment depends slightly on $R e$ for $R e>5 \times 10^{5}$. This phenomenon may be explained by the different separation points in turbulent flow and laminar flow. When $R e$ becomes larger, the flow at the upstream of the weir changes from laminar flow to turbulent flow, the separation point moves down along the surface of the weir. So the reattachment point moves towards the upstream. When $R e$ is large enough, the flow at the upstream of the weir becomes a full developed turbulent flow, the separation point does not change any longer.

For surface wave and plunging condition, the water level of the downstream is lower than that of the upstream with the increase of $F r_{\mathrm{u}}$ or $D / H$. The potential energy of the flow can overcome reverse pressure gradient, the separation point will move down, and the reattachment point moves to the upstream. The effects of the ratio of water depth at downstream to that at upstream $h / H$ on the reattachment point is shown in Fig.5. Figure 
5 shows that the position of the reattachment changes slowly with $h / H$ for $h / H>0.86$, and the reattachment point moves to the weir suddenly when $h / H<0.86$. This condition corresponds to the critical point that surface wave turns to plunging jet.

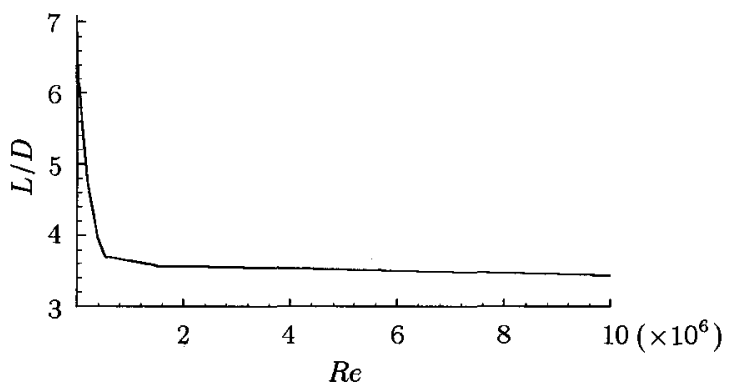

Fig.4 The relationship of the position of the reattachment with $R e . F r_{\mathbf{u}}=$ $0.075, D / H=0.67$

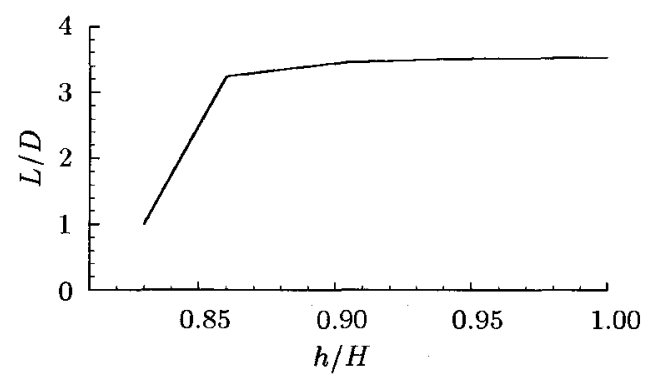

Fig.5 The relationship of the position of the reattachment point with $h / H$. $R e=1.5 \times 10^{6}$

In order to calculate the position of the reattachment point accurately, the effect of the mesh size is discussed. The results of different mesh size are compared with the experiments. Under a coarse mesh condition (the total number of the grid notes is about 7500 ), the numerical results and the experiments have a discrepancy about $12 \%$. Under a refined mesh condition (the total number of the grid notes is more than 15000 ), the discrepancy of the numerical results and the experiments is less than $5 \%$, and the discrepancy caused by the effects of mesh size is less than $3 \%$.

\subsection{Velocity Profiles and Bottom Shear Stress}

The velocity profile and the bottom shear stress at the downstream of the weir are obtained by numerical simulation and experiment. These results are shown in Fig.6 and Fig.7, respectively. Figure 6 and Fig.7 show that the numerical results agree well with the experimental data.

For surface jet and surface wave, there is a reverse flow between the reattachment point and the weir (see in Fig.6(a)). The bottom shear stress in this region is higher than that at the far downstream (see in Fig.7(a)). The maximum bottom shear stress $\tau$ is at $X=2 D \sim 3 D$ ( $X$ is the distance from the center of the weir), and the value can reach

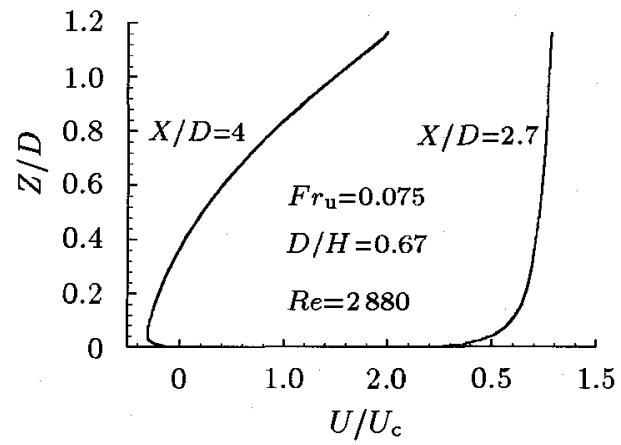

(a) Surface jet

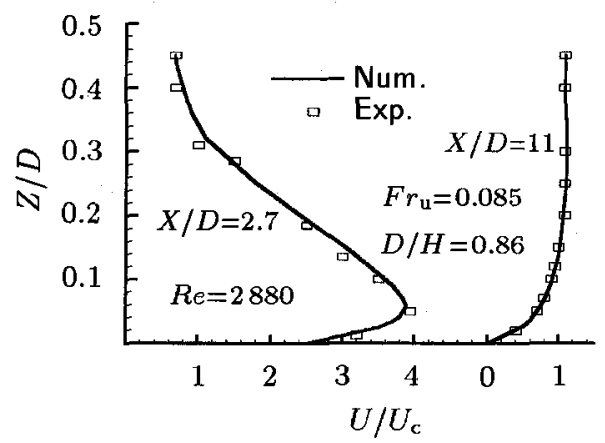

(b) Plunging condition

Fig.6 The velocity profile at the downstream of the weir. $U_{c}$ : the mean flow velocity 


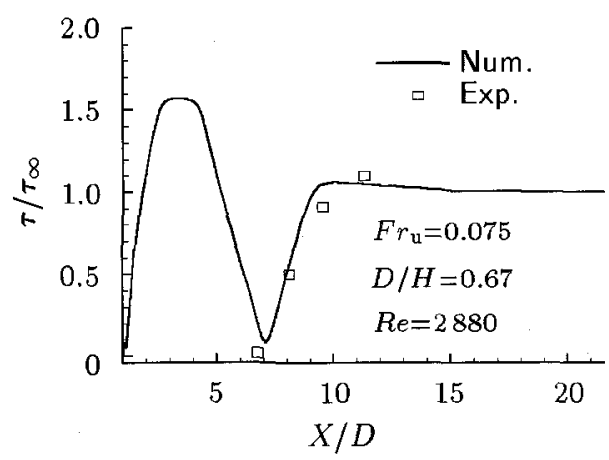

(a) Surface jet

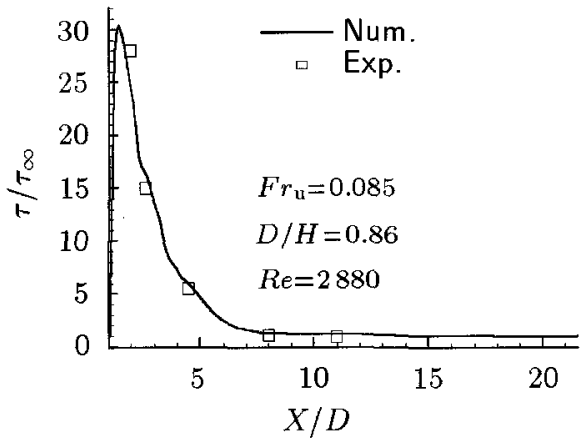

(b) Plunging condition

Fig.7 The bottom shear stress at downstream

$1 \tau_{\infty} \sim 2 \tau_{\infty}\left(\tau_{\infty}\right.$ is the bottom shear stress at far downstream). Near the reattachment point the bottom shear stress approaches to zero. For $X>10 D$, the difference of $\tau$ and $\tau_{\infty}$ is less than $5 \%$. So the region of the scour at the downstream is less than $10 D$. For the plunging jet, the high velocity stream near the bottom leads to a very high value of the bottom shear stress near the weir. The maximum $\tau$ is at $X=1.5 D \sim 2 D$, and the value is more than $10 \tau_{\infty}$. The bottom shear stress decreases as $X$ increases (see in Fig. $7(\mathrm{~b})$ ). The region of scour can reach 2 or 3 times of that under the surface jet and the surface wave conditions.

\section{CONCLUSION}

The flow over a semicircular weir was investigated numerically and experimentally. The VOF method with a geometric reconstruction approach and the unstructured mesh employed in the numerical simulation are able to simulate large deformation of free surface and improve the accuracy of the calculation.

The experiments show four types of flow patterns: surface jet, surface wave, plunging jet, hydraulic jump and the corresponding range of the parameters $F r_{\mathrm{u}}, h / H$ and $D / H$. The numerical results of three types of the flow patterns compare well with the experiments.

The position of reattachment has been studied. For surface jet, the position of reattachment point is predominated by $R e$. When $R e<5 \times 10^{5}$, the position of the reattachment point moves towards the upstream with increase of $R e$. When $R e>5 \times 10^{5}$, the flow at the upstream of the weir becomes a full developed turbulent flow, the position of the reattachment changes little. For surface wave and plunging condition, the distance from the position of the reattachment to the center of the weir decreases as the water level at the downstream of the weir is low. When the flow pattern changes from the surface wave to the plunging condition, the reattachment point moves to the weir suddenly.

The velocity profile and the bottom shear stress have been obtained by numerical simulations. The results of numerical simulations agree very well with experimental data. For surface jet and surface wave, the maximum shear stress can reach $1 \tau_{\infty} \sim 2 \tau_{\infty}$, the region of the scour at the downstream is less than $10 D$. For plunging jet, the maximum shear stress is more than $10 \tau_{\infty}$, The region of scour can reach 2 or 3 times of that under the surface jet and the surface wave conditions. 


\section{REFERENCES}

1 Lamb H. Hydrodynamics. Dover, 1945

2 Forbes LK, Schwartz LW. Free-surface flow over a semicircular obstruction. J Fluid Mech, 1982, 114: $299 \sim 314$

3 Dias F, Vanden Broeck JM. Open channel flow with submerged obstacles. J Fluid Mech, 1989, 206: $155 \sim 170$

4 Hirt CW, Nichols BD. Volume of fluid (VOF) method for the dynamics of free boundaries. $J$ Comput Phys, 1981, 39: 201 225

5 Liu QC, Chen CT. Numerical study of turbulence characteristics in hydraulic jumps. Chinese J Hydraulics, 1993, 1: 1 10

6 Bradford SF. Numerical simulation of surf zone dynamics. $J$ Waterway Port Coastal and Ocean Eng, 2000, 126: 1 13

7 Fadda D, Raad PE. Open channel flow over submerged obstructions: an experimental and numerical study. J Fluids Eng, 1997, 119: 906 910

8 Rhie CM, Chow WL. Numerical study of the turbulent flow past an airfoil with trailing edge separation. AIAA, 1983, 21: 1525 1532 